\title{
Rational Trust in Resilient Health Systems
}

Sonja Kristine Kittelsen, Centre for Development and Environment, University of Oslo, Oslo, Norway Vincent Charles Keating, Center for War Studies, University of Southern Denmark, Odense, Denmark

This is a pre-print version of an article published by Oxford University Press in the journal Health Policy and Planning.

\section{Abstract}

The 2014-2015 Ebola epidemic in West Africa highlighted the significance of trust between the public and public health authorities in the mitigation of health crises. Since the end of the epidemic, there has been a focus on what scholars and practitioners have called resilient health systems, which many see as an important precondition for successfully combatting future outbreaks. While trust has been acknowledged as a relevant component of health systems resilience, we argue that a more serious theoretical engagement in underlying models of trust is needed in the literature. This paper takes a first step to fill this gap by demonstrating how the health systems resilience literature appeals to rational models of trust in the construction of resilient health systems, and how currently unconsidered assumptions in this model cast doubt on the effectiveness of strategies to generate trust, and therein resilience, during acute public health emergencies.

Keywords: trust; resilience; health systems; Ebola; health emergencies

\section{Introduction}

The concept of health systems resilience has gained considerable traction in the years following the Ebola epidemic in West Africa in 2014-2015. The epidemic exposed the fragility of the health systems in the three Ebola-affected countries, Guinea, Liberia, and Sierra Leone, and underscored the centrality of a strong health system in effectively detecting and containing outbreak events. Limited health workforce capacity, poor health infrastructure, inadequate surveillance and response systems, and weak community engagement in all three countries hindered the ability to manage the virus (Shrivastava et al., 2016: 105; Naimoli et al., 2018: 4). In the wake of the epidemic, several articles and reports have argued for the need 
to strengthen countries' health systems capacities to be better able to detect and respond to outbreak events, while also providing safe and effective health services (Heymann et al., 2015: 1884; Writh et al., 2015). This has included a focus on strengthening countries' health systems to become more 'resilient' in the face of future health risks (Kamal-Yanni, 2015; GHRF Commission (Commission on a Global Health Risk Framework for the Future), 2016; Govindaraj et al., 2018).

A common thread throughout the resilience literature is a recognition of the centrality of trust in resilient health systems. Indeed, lack of trust between community members and public health authorities in the three Ebola-affected countries hindered response efforts, leading a number of scholars to stress the importance of trust to both the functioning of health systems and the mitigation of adverse public health events (Huff, 2015; Shrivastava et al., 2016). Nevertheless, while "trust" is identified as both needed and even essential to good outcomes in health crises, there is very little sustained theoretical consideration over exactly what trust is and, consequently, how it can be built - particularly with respect to the precise nature of the causal relationships between alleged trust-building activities and trusting outcomes.

The purpose of this paper is to take a first step in rectifying this problem by demonstrating some of the conceptual problems underpinning the literature's engagement with trust, engaging specifically with scholars that implicitly use rational trust models when putting forward strategies for increasing resilience. Rational models of trust are based on the idea that trust can be built through ongoing, positive exposure to health systems. They assume that, if exposure to poor health services creates distrust amongst members of the population (Aitsi-Selmi et al., 2015; Cancedda et al., 2016; GHRF Commission (Commission on a Global Health Risk Framework for the Future), 2016; Bitton et al., 2017) - a claim that is almost certainly true then the reverse must also be true: the consistent provision of effective health services will foster trust in the health system, which can then be "cashed-in" during health crises like Ebola.

We suggest, however, that there are three unconsidered assumptions underlying this argument that need to be empirically explored. These concern: (1) the nature of the payoffs to engagement with a health system in a health crisis; (2) the nature of trust as a generalized or specific phenomenon; and (3) the problems associated with the use of hedging as a coping strategy when trust is not completely conferred in a health system. To make any claim that positive health experiences will translate into trust during health crises, these assumptions, must be at a minimum considered within any study of trust.

To make our argument, we begin by providing an overview of the concept of health systems resilience and the role that trust plays within it. Then, drawing from theories of trust in sociology and political science, we put forward three limitations of rational models of trust found within the health systems resilience literature. In putting forward our argument, we hope that this paper serves as a call for a more 
theoretically-informed engagement with trust in the health systems resilience literature, so that scholars can provide causal clarity over exactly how and why certain types of interventions will be effective in building and sustaining trust.

\section{Background: Health systems resilience and the centrality of trust}

The concept of health systems resilience has gained increased currency amongst academics and policymakers alike, often in reference to the ability of a health system to anticipate and respond effectively to external shocks. Central to this approach is a focus on the ability of health systems to identify and detect a broad range of challenges, whether acute or more chronic in nature, and adjust accordingly without compromising their core functions (Kruk et al., 2015: 1910; Barasa et al., 2017: iii93; Ling et al.: iii41; Hanefeld et al.: 356$)$. A theme that cuts across the health systems resilience literature, moreover, is the importance of trust in fostering resilience.

Trust is presented in the majority of the literature as a precondition for resilience: it is a factor that determines the demand or utilisation of health services, both on an everyday basis and during a crisis, and as an important factor in the mitigation of a health emergency (Ozawa et al., 2016: 132; Kruk et al., 2017). Johanna Hanefeld and colleagues (2018: 365) have argued, for example, that 'the level of trust in public (including health) institutions may be key to the ability of health systems to withstand shocks. Trust affects the relationship between the people and the health system, including whether and how people access and use health services, what information citizens are willing to share with the government and whether health workers are responsive to local needs.' Similarly, Kruk et al. (2017) have identified trust in government and the health system as not only an essential component to health service delivery, but also to effective government messaging in times of crisis.

A strong health system, in turn, is recognised as an important component in fostering trust between community members and public (health) authorities. This includes the ability of the health system to respond quickly during a health emergency. The barriers to accessing healthcare for both Ebola and more common conditions during the 2014-2015 Ebola epidemic in West Africa, for example, served to undermine trust in the health system in the three Ebola-affected countries and hinder response efforts (Kieny \& Dovlo 2015 , 91). The relationship between trust and health systems resilience is thus recognised as mutually reinforcing: trust is key to building a resilient health system, while a strong health system is key to fostering trust. However, as the next section demonstrates, there are three important problems that, because of a general lack of engagement in trust theory, have yet to be considered. 


\section{Rational trust models in health systems resilience literature}

In rational models of trust, trust is recognised as primarily, if not solely, a calculative exercise that involves gathering information about other actors as a means of predicting their likely future behaviour. To determine whether it is a good decision to trust the health system with his or her care, an individual must make a calculation. As James Coleman (1990: 99) put it, trust is conferred 'if the chance of winning, relative to the chance of losing, is greater than the amount that would be lost (if he loses), relative to the amount that would be won (if he wins).' This means that the central variables surrounding any decision to trust a health system are threefold. There are: (1) the particular positive payoffs associated with the health system doing what is expected of it; (2) a series of direct and opportunity costs involved in engaging with the health system, including the potential failure of the health system to cure the illness or make things worse; and (3) a probability that the health system will provide the positive payoffs for the individual. If engaging with the health system yields a positive expected value, then according to rational models, trust is conferred. If it results in a negative expected value, then trust is not conferred.

According to the rational model, an individual's decision to trust in a health system will be structured by the amount of information that he or she has about the character and interests of the health system. The more information an individual has about a health system, the greater the likelihood that they will correctly identify that the health system is (or is not) trustworthy. This process is sometimes modelled using a Bayesian approach (Hardin, 1993: 155; Kydd, 2005: 19), whereby an initial estimate is made of trustworthiness, which is then revised upwards or downwards depending on whether new information is gained that is positive or negative about the character and interests of the health system.

Within the health systems resilience literature, this understanding of trust corresponds with what might be called a technical model of health system resilience. Here trust is established through consistent positive experiences with the health system. Much of the literature stresses the need to strengthen health worker capacity through training them with the right competencies, ensuring that primary clinics have adequate staffing and support, or focusing on ensuring that there are proper managers or information systems in place prior to a potential outbreak (Kieny and Dovlo, 2014: 92; Dhillon and Yates, 2015: e435; Kirigia et al., 2015: 8; Gostin et al., 2016: 1451; Perry et al., 2016: 552; Kruk et al., 2017: 35). These measures help to create a high-quality service environment that will gain the public's trust and increase resilience (Kruk et al., 2015: 1910; World Health Organization, 2015: 11). Similarly, Kruk et al. (2015: 1911) as well as Ranu S. Dhillon and Robert Yates (2015: e435) have pointed to how investment in universal health coverage can help to build trust with communities otherwise disaffected from health care. 
In all cases, these solutions are driven by an underlying rational model of trust because they presume that having constant positive experiences with the health system will build trust. These positive experiences can then be "cashed-in" in the form of increased trust during an acute health crisis, strengthening resilience. However, while dysfunctional health systems will almost certainly lead to increased distrust, there are three underlying assumptions in the rational model that need to be considered prior to making the claim that positive experiences with the health system will automatically increase public perceptions of trust in the system during an acute health event.

\section{Assumption 1: Similar benefits and losses}

The first assumption underpinning the rational trust model is that the benefits and losses in accessing health services during times of crisis are roughly proportional to normal times. If this is the case, then the increased level of trustworthiness established during times of calm would directly transfer to times of crisis, contributing to the resilience of the system. This is not a straightforward claim, however. During a health crisis, the perceived potential loss might increase significantly since the crisis might suddenly involve the potential for imminent suffering and death, while the benefits of the health system might be seen as reduced - particularly if the crisis involves a novel illness that is not easily treated.

This point reflects much of the literature that stresses how health crises such as epidemics can produce fear amongst the general population (De Roo et al., 1998; Leppin and Aro, 2009: 12-18; Dhillon and Yates, 2015: 2015) which, during previous epidemic outbreaks in states with well-functioning health systems, resulted in significant reductions in the public's care-seeking behaviour (Chang et al., 2004). This was no different with Ebola. Indeed, as David Nabarro and Chadia Wannous (Nabarro and Wannous, 2016: 228) put it, 'In the Ebola outbreak in West Africa, fear and panic led to distrust and may have exacerbated the spread of the disease and its subsequent impact on social cohesion.'

Thus, while having an increased perception of health systems trustworthiness during times of calm will be of some benefit in the event of a health crisis, depending on the nature of the health crisis, any trust gains made might be swept away by a massive increase in perceived losses, inhibiting individuals from trusting the health system exactly at the time when trust is needed. Any strategy based on rational trust must therefore demonstrate that the loss-perception differences between periods of calm and crisis is not significantly great enough to undermine the trust-building that occurred before the crisis. 


\section{Assumption 2: Generalized trust}

The second assumption focuses on the other major variable in the rational trust model - the perception of trustworthiness. Here the question concerns whether or not perceptions of trustworthiness are generalised or specific. Much of the health systems resilience literature implies that trust is generalised, that is, that the public's level of trustworthiness for the health system will be the same for all possible interactions: $X$ simply trusts $Y$ at level t. For instance, within Kruk et al.'s framework for health system resilience, trust is identified as a product of a diverse health system, fostered through the consistent provision of health services: 'In times of calm, systems that address diverse health needs will increase the number and quality of people's interactions with the health system, enhancing public trust and enabling more rapid recognition of a new threat, realising the resilience dividend' (Kruk et al., 2015: 1911). There is an implicit idea here that the trustworthiness gained through positive interactions with the health system during times of calm will unproblematically transfer over to the time of crisis.

However, it could be that a better model would be one of specific trust, where actors do not simply trust each other, but trust each other to do specific things (and not other things) (Levi and Stoker, 2000: 476). To put it simply, specific trust models argue that $X$ trusts $Y$ to do $Z$; $X$ does not simply trust $Y$. If we consider the issue of building trust from a specific trust perspective, then the primary question would be: if the public grows to trust the health system to deliver on a particular $Z$, that is, routine health services, would the public have the same level of trustworthiness if we introduce a new $Z$ such as a public health crisis? This cannot simply be taken for granted. Perceived capability and competence will be part of any rational decision to engage with a health system, and regular health service delivery is not the same as health service delivery in times of crisis. So, while sustained positive interactions might increase trust during normal times, it does not necessarily follow that it will also lead to a similar level of trustworthiness in what might be considered a distinct domain - crisis management.

\section{Assumption 3: Clear attribution of success}

The third problem is not directly related to the variables in the rational trust model, but complicates the way that individuals gather information to judge trustworthiness. Under the rational model with every interaction that results in a positive experience, the trustworthiness estimator increases under the Bayesian model, making it more likely an individual will engage with the health system. This idea that trust is about engagement or lack of engagement is central to the literature. For instance, Lucy Gilson (2005: 1381) has noted that 'the level of trust in the patient-provider relationship influences health-seeking behaviour in a low-income urban setting.' Ozawa et al. (2016: 132) also notes that 'Trust is a critical 
determinant of demand for services, and plays an essential role in user-provider interactions, which are at the center of the healthcare system.'

Structuring the conversation around engagement or non-engagement, however, misses out on an important element of how people respond to risk. Only in some situations will the available options be either to engage or not to: in other cases individuals will choose to hedge against the perceived risk (Keating and Ruzicka, 2014). In choosing to hedge, members of the public reduce their dependence on, and vulnerability to, a health system that is not fully trusted, by seeking out alternative means of treatment simultaneously. Not fully trusting the system means that individuals will interact with the health system and, hedging their bets, take the additional costs to interact with alternative sources as well.

But this rational response to hedge in the face of risk then complicates the otherwise straightforward story about the link between information gathering and perceived trustworthiness, and poses a problem for the construction of trust between the system and members of the public. If a member of the public has a health problem and has it successfully treated, but hedged against the risk of it not working by going to an alternative source simultaneously, then with whom is trust conferred? There is no automatic necessity that it will be the public health system. Thus, even if good service is provided, the existence of hedging activities means that the effect of that good treatment on trustworthiness might not be as high as expected.

\section{Conclusion}

The purpose of this paper has been to demonstrate the importance of theoretically engaging with sociological concepts through our focus on the implicit rational models of trust that are often found in the resilience literature. While, there is no question that negative experiences with the health system prior to a crisis would undermine trust, we argue that there are three underlying problems with the rational trust logic that, at the very least, require further consideration if these models are to be used to build resilience for acute health events.

Ensuring that these capabilities exist before a crisis, therefore, is unto itself no guarantee of success with respect to trust-building unless the implications of the logic underpinning the rationalist model of trust are thoroughly thought through. Echoing what Fred P. Martineau (2016: 308) has previously stated, 'the ability to provide effective clinical care is a necessary - but insufficient - component of the trustworthiness of a health system.' We hope that this paper serves as a first step in a more open consideration of the underlying trust models that are used in the health systems resilience literature, and a more critical eye on 
exactly what the causal claims of these models are, and whether they stand up to scrutiny both theoretically and empirically.

\section{Bibliography}

Aitsi-Selmi A, Egawa S, Sasaki H, et al. (2015) The Sendai Framework for Disaster Risk Reduction: Renewing the Global Commitment to People's Resilience, Health, and Well-being. International Journal of Disaster Risk Science 6: 164-176.

Barasa EW, Cloete K and Gilson L. (2017) From bouncing back, to nurturing emergence: reframing the concept of resilience in health systems strengthening. Health Policy and Planning 32: iii91-iii94.

Bitton A, Ratcliffe HL, Veillard JH, et al. (2017) Primary Health Care as a Foundation for Strengthening Health Systems in Low- and Middle-Income Countries. Journal of General Internal Medicine 32: 566571.

Cancedda C, Davis SM, Dierberg KL, et al. (2016) Strengthening Health Systems While Responding to a Health Crisis: Lessons Learned by a Nongovernmental Organization During the Ebola Virus Disease Epidemic in Sierra Leone. J Infect Dis 214: S153-s163.

Chang H-J, Huang N, Lee C-H, et al. (2004) The Impact of the SARS Epidemic on the Utilization of Medical Services: SARS and the Fear of SARS. American Journal of Public Health 94: 562-564.

Coleman J. (1990) Foundations of Social Theory, Cambridge, MA: The Belknap Press of Harvard University Press.

De Roo A, Ado B, Rose B, et al. (1998) Survey among survivors of the 1995 Ebola epidemic in Kikwit, Democratic Republic of Congo: their feelings and experiences. Trop Med Int Health 3: 883-885.

Dhillon RS and Yates R. (2015) Building back better: priorities for Ebola-affected countries. The Lancet Global Health 3: e435-e436.

GHRF Commission (Commission on a Global Health Risk Framework for the Future). (2016) The Neglected Dimension of Global Security: A Framework to Counter Infectious Disease Crises.

Gilson L. (2005) Editorial: building trust and value in health systems in low- and middle-income countries. Social Science \& Medicine 61: 1381-1384.

Gostin LO, Mundaca-Shah CC and Kelley PW. (2016) Neglected dimensions of global security: The global health risk framework commission. JAMA 315: 1451-1452.

Govindaraj R, Herbst CH and Clark JP. (2018) Strengthening Post-Ebola Health Systems: From Response to Resilience in Guinea, Liberia, and Sierra Leone, Washington, D.C.: World Bank.

Hanefeld J, Mayhew S, Legido-Quigley H, et al. (2018) Towards an understanding of resilience: responding to health systems shocks. Health Policy Plan 33: 355-367.

Hardin R. (1993) The street-level epistemology of trust. Analyse \& Kritik 14: 152-176.

Heymann DL, Chen L, Takemi K, et al. (2015) Global health security: the wider lessons from the west African Ebola virus disease epidemic. The Lancet 385: 1884-1901.

Huff AR. (2015) Ebola and Lessons for Development. IDS Practice Paper in Brief 16. Brighton: Institute for Development Studies.

Kamal-Yanni. (2015) Never Again: Building resilient health systems and learning from the Ebola crisis. Oxfam Briefing Paper. Cowley, Oxford: Oxfam International.

Keating VC and Ruzicka J. (2014) Trusting Relationships in International Politics: No Need to Hedge. Review of International Studies 40: 753-770. 
Kieny MP and Dovlo D. (2014) Beyond Ebola: a new agenda for resilient health systems. The Lancet 385: 9192.

Kirigia JM, Masiye F, Kirigia DG, et al. (2015) Indirect costs associated with deaths from the Ebola virus disease in West Africa. Infectious Diseases of Poverty 4: 45.

Kruk ME, Ling EJ, Bitton A, et al. (2017) Building resilient health systems: a proposal for a resilience index. BMJ: British Medical Journal 357: 2323.

Kruk ME, Myers M, Varpilah ST, et al. (2015) What is a resilient health system? Lessons from Ebola. Lancet 385: 1910-1912.

Kydd A. (2005) Trust and mistrust in international relations, Princeton: Princeton University Press.

Leppin A and Aro AR. (2009) Risk Perceptions Related to SARS and Avian Influenza: Theoretical Foundations of Current Empirical Research. International Journal of Behavioral Medicine 16: 7-29.

Levi M and Stoker L. (2000) Political Trust and Trustworthiness. Annual Review of Political Science 3: 475507.

Ling EJ, Larson E, Macauley RJ, et al. (2017) Beyond the crisis: did the Ebola epidemic improve resilience of Liberia's health system? Health Policy Plan 32: iii40-iii47.

Martineau F. (2016) People-centred health systems: building more resilient health systems in the wake of the Ebola crisis. International Health 8: 307-309.

Nabarro D and Wannous C. (2016) The Links Between Public and Ecosystem Health in Light of the Recent Ebola Outbreaks and Pandemic Emergence. EcoHealth 13: 227-229.

Naimoli JF, Saxena S, Hatt LE, et al. (2018) Health system strengthening: prospects and threats for its sustainability on the global health policy agenda. Health Policy Plan 33: 85-98.

Ozawa S, Paina L and Qiu M. (2016) Exploring pathways for building trust in vaccination and strengthening health system resilience. BMC Health Services Research 16: 639.

Perry HB, Dhillon RS, Liu A, et al. (2016) Community health worker programmes after the 2013-2016 Ebola outbreak. Bulletin of the World Health Organization 94: 551-553.

Shrivastava SR, Shrivastava PS and Ramasamy J. (2016) Ebola outbreak in West Africa: Bridging the gap between the public health authorities and the community. Iranian Journal of Nursing and Midwifery Research 21: 105-106.

World Health Organization. (2015) 2015 WHO Strategic Response Plan: West African Ebola Outbreak. Geneva.

Writh S, Hanna L and Mailfert M. (2015) A Wake-Up Call; Lessons from Ebola for the world's health systems. London: Save the Children. 